CLINICAL ALERT

\title{
Chronic pancreatitis, depression and substance use disorders: A not uncommon combination
}

\author{
C Y Jeppe, C P Szabo, M D Smith
}

Cara Yvonne Jeppe is a research facilitator in the Hepatopancreatobiliary Unit in the Department of Surgery at Chris Hani Baragwanath Academic Hospital, Johannesburg, South Africa. She has a professional background as a medical technologist and her interests include social determinants of disease and the outcomes of their surgical management. Prof. Christopher Paul Szabo is Head of the Department of Psychiatry, Faculty of Health Sciences, University of the Witwatersrand, Johannesburg, and Head of the Department of Psychiatry at Charlotte Maxeke Johannesburg Academic Hospital, Johannesburg. His areas of special interest include eating disorders and ethics. Adjunct Prof. Martin Derrick Smith is Head of the Department of Surgery, Faculty of Health Sciences, University of the Witwatersrand, and Head of the Department of Surgery at Chris Hani Baragwanath Academic Hospital. He has a special interest in hepatopancreatobiliary surgery.

Corresponding author: C P Szabo (christopher.szabo@wits.ac.za)

Chronic pancreatitis (CP) is a progressive and debilitating disease. A potentially important consideration is the relationship between $\mathrm{CP}$, depression and substance use disorders, which seems to be circular and multiplicative. Pain management is a critical component of intervention, and it would seem that in the context of chronic illness this requires a biopsychosocial approach aiming for a tailored intervention that strikes an appropriate therapeutic balance.

S Afr Med J 2015;105(3):179-180. DOI:10.7196/SAMJ.8885

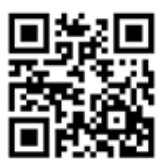

Chronic pancreatitis $(\mathrm{CP})$, a progressive and debilitating disease that usually presents with pain, is frequently attributed to alcohol misuse. Patients generally present for treatment in their fourth decade after recurring absenteeism and job loss; some have become (or have always been) indigent. There is a need for prevention. A potentially important consideration is the relationship between $\mathrm{CP}$, depression and substance use disorders (SUDs), which seems to be circular and multiplicative.

\section{Pain, depression and substance abuse}

Compared with people in good health, 'depression is approximately two to three times more common in people with a chronic physical health problem. ${ }^{[1]}$ In their article discussing the neurobiology and clinical presentation of pain and its synergies with SUD, Savage et al. ${ }^{[2]}$ stated: 'Pain and substance abuse co-occur frequently, and each can make the other more difficult to treat'; 'each may reinforce components of the other'; and 'depression is the most common psychiatric symptom seen among pain patients'. They found that clinicians' fear that patients might develop SUD with opioid treatment for pain compromised effective treatment. ${ }^{[2]}$ Mavandadi et al. ${ }^{[3]}$ found that pain 'may hinder or minimize treatment-related improvements in depressive symptoms'. Concerns about substance dependence resulting from opioid use may therefore contribute to inadequate pain relief and also lead to suboptimal response to treatment of depression, while potentially contributing towards depressive symptoms. The interplay of these factors has clear implications for the management of CP and calls for a multidisciplinary approach to optimise outcome.

\section{Diagnosis of depression}

Depression is frequently undetected and untreated. While poor socioeconomic status can predispose people to depression, which can begin in childhood or adolescence, it can also make diagnosis and treatment less likely. Other risk factors for a depressive disorder include alcohol abuse, stressful life events, adverse childhood experiences, job loss, close personal relationship problems, poverty, and a family history of depression. Severe pain is a major contributor to depression, and pain amelioration can improve collaborative care interventions for depression.

In 1985 David Goldberg ${ }^{[4]}$ identified five main reasons why patients failed to have their psychiatric conditions diagnosed by physicians: they would not be asked directly about their psychological conditions; only somatic symptoms would be discussed; privacy during consultations would be lacking; there would be an exclusive focus on organic causes of symptoms; and physicians lacked confidence in their ability to make psychiatric assessments. Ideally, consultants should have knowledge of the culture of origin of patients with depression, within a holistic biopsychosocial approach to disease management. Knowledge of the social environment of such patients is critical for determining what measures can be taken to improve their circumstances and thus contribute to amelioration of depression. Social workers from the community are well placed to facilitate such measures.

\section{Depression and physical illness}

Objective evidence on the incidence and severity of depression in combination with chronic physical health problems is not available. ${ }^{[1]}$ Somatic symptoms of the latter can occur with, and mask, the symptoms of the former, making distinction between the two problematic. ${ }^{[1]}$ The simultaneous presence of both increases the likelihood and extent of functional, social and occupational disabilities, and may lead to shorter life expectancies. Depression worsens medical prognosis and may play an aetiological role in SUD and CP. In 2000, DiMatteo et al. ${ }^{[5]}$ found that depressed patients with physical illness were three times more likely to be non-compliant with treatment recommendations. Although active treatment for depression may have a lesser beneficial effect for severe pain and may not improve length of survival, it improves quality of survival.

\section{Chronic pancreatitis, substance abuse and depression}

Depression and low self-esteem arising from poor education and unemployment are associated with high-risk behaviours such as 
substance abuse, while SUD can be both the cause and effect of depression. Wehler et al. ${ }^{[6]}$ lamented the lack of knowledge about poor mental health in patients with CP and noted that a substantially increased risk for onset of major depressive disorders arises from alcohol abuse. Such a causal link has also been suggested by Fergusson et al. ${ }^{[7]}$ Drug addiction has been cited as a complication of depressive disorders, and dependence on opioids can result from their use as prescribed treatment for CP pain. Unemployment may be a predictor of abuse of prescription opioids and vice versa, while both increase dependence on disability grants.

\section{Chronic pancreatitis, surgery and pain management}

The Frey procedure (local resection of the pancreatic head with lateral pancreaticojejunostomy) undertaken at the Hepatopancreatobiliary (HPB) Unit in the Department of Surgery at Chris Hani Baragwanath Academic Hospital, Johannesburg, South Africa, between 2002 and 2009 improved quality of life and functioning and reduced symptoms in 32 patients with painful $\mathrm{CP} \cdot{ }^{[8]}$ The median duration of pain before surgery was 72 months (range 10 - 264). There was no significant correlation between preoperative pain duration and the pain score or pain intensity of the 32 patients. The traditional view that with time there is 'burn-out' of pain has been invalidated, ${ }^{[8-10]}$ and deferment of surgery in anticipation of future 'burn-out' is therefore not appropriate. Rather, deferment of surgery increases the risk of dependence on opioids.

In a later unpublished study from the same authors, 35 patients treated between 2009 and 2012 answered a structured interview before and after the Frey procedure in the HPB Unit. All patients answered questions relating to hopelessness and pain, as measured on a visual analogue scale from 0 to 10 . The use of prescribed opioids was recorded at both interviews. The duration of postoperative follow-up was a mean of 27.5 months (standard deviation 24.9). Before surgery, $68.6 \%$ of the patients felt hopeless, $100 \%$ had a pain score of $\geq 5$, and $38.2 \%$ were using prescribed opioids. After surgery, at the last recorded visit, $34.3 \%$ felt hopeless, $54.5 \%$ had pain $\geq 5$, and there was an increase in the use of prescribed opioids to $64.7 \%$ of the sample. The increasingly liberal prescribing of opioids has raised concern about dependence and deleterious side-effects. ${ }^{[1]}$ Misuse of opioids for the relief of depression can lead to behaviours that exaggerate pain in order to increase the amount of opioids prescribed. In a rare study on interventions for alcohol abuse in patients with alcohol use disorders and painful pancreatitis, a reduction in alcohol consumption and a decreased need for analgesics was achieved by a multidisciplinary group of pancreatic surgeons and addiction and pain specialists. ${ }^{[12]}$

\section{Conclusion}

The use of adequate analgesia is a key component in preventing depressive symptoms, but may promote substance dependence and abuse. The clinician is therefore caught in a double bind. Pain management in the context of chronic illness requires a biopsychosocial approach aiming for a tailored intervention that strikes an appropriate therapeutic balance.

Surgeons, pain and addiction clinicians working in collaboration with nurses, social workers, psychologists and psychiatrists can contribute to improved quality of life for patients with $\mathrm{CP}$, and raise professional and public awareness at all levels of healthcare.

\footnotetext{
1. National Collaborating Centre for Mental Health. Depression in adults with a chronic physical health problem. In: Depression in Adults with a Chronic Physical Health Problem: The NICE Guideline on Treatment and Management. Chap. 2. British Psychological Society and Royal College of Psychiatrists 2010:15-27. hom 2010:15-27. http://www.nice.org.uk/guidance/cg91/evidence/cg91-depression-with-a-chronic-physical-
health-problem-full-guideline2 (accessed 30 January 2015). health-problem-full-guideline2 (accessed 30 January 2015).

Savage SR, Kirsh KL, Passik SD. Challenges in using opioids to treat pain in persons with substance use disorders. Addict Sci Clin Pract 2008;4(2):4-25. [http://dx.doi.org/10.1151/ascp08424]

3. Mavandadi S, Ten Have TR, Katz IR, et al. Effect of depression treatment on depressive symptoms in older adulthood: The moderating role of pain. J Am Geriatr Soc 2007;55(2):202-211. [http://dx.doi. org/10.1111/j.1532-5415.2007.01042.x]

4. Goldberg D. Identifying psychiatric illness among general medical patients. BMJ 1985;291(6489):161162. [http://dx.doi.org/10.1136/bmj.291.6489.161]

5. DiMatteo MR, Lepper HS, Croghan TW. Depression is a risk factor for noncompliance with medical treatment: Meta-analysis of the effects of anxiety and depression on patient adherence. Arch Intern Med 2000;160(14):2101-2107. [http://dx.doi.org/10.1001/archinte.160.14.2101]

6. Wehler M, Nichterlein R, Fischer B, et al. Factors associated with health-related quality of life in chronic pancreatitis. Am J Gastroenterol 2004;99(1):138-146. [http://dx.doi.org/10.1111/j.15720241.2004.04005.x]

7. Fergusson DM, Boden JM, Horwood J. Tests of causal links between alcohol abuse or dependence and major depression. Arch Gen Psychiatry 2009;66(3):260-266. [http://dx.doi.org/10.1001/ archgenpsychiatry.2008.543]

8. Jeppe CY, Becker P, Smith MD. Post-Frey procedure quality of life in South African patients with painful chronic pancreatitis. J Pancreas (Online) 2013;14(1):21-30. [http://dx.doi.org/10.6092/1590$577 / 933$

9. Mullady DK, Yadav D, Amann ST, et al. Type of pain, pain-associated complications, quality of life, disability and resource utilization in chronic pancreatitis: A prospective cohort study. Gut 2011;60(1):77-84. [http://dx.doi.org/10.1136/gut.2010.213835]

10. Liu LS, Shenoy M, Pasricha PJ. Substance P and calcitonin gene related peptide mediate pain in chronic pancreatitis and their expression is driven by nerve growth factor. J Pancreas (Online) 2011;12(4):389-394.

1. Sehgal N, Colson J, Smith HS. Chronic pain management with opioid analgesics: Benefits versus harms of long-term therapy. Expert Review of Neurotherapeutics 2013;13(11):1201-1220. [http://dx.doi.org 110.1586/14737175.2013.846517]

12. Lang MB, Segersvard R, Grundsten $M$, et al. Management of alcohol use disorders in patients with chronic pancreatitis. J Pancreas (Online) 2012;13(6):654-659. [http://dx.doi.org/10.6092/15908577/1037]
}

Accepted 8 September 2014.

\section{This month in the $S A M J$...}

Greta Dreyer* is a gynaecological oncologist at Steve Biko Academic Hospital, Pretoria, where she heads the clinical and research units in the Department of Obstetrics and Gynaecology at the University of Pretoria. She is a founder member of the South African HPV Advisory Board and its research fund and of the University of Pretoria and Netcare Familial Cancer Centre, and established the Southern African Journal of Gynaecological Oncology, of which she is still Editor-in-Chief. She holds a doctorate in basic research and clinical cancer genetics for work done on the PTEN gene in gynaecological cancer. Her research efforts in oncology are currently mostly aimed at the control of lower genital tract cancer via immunisation and screening, the recognition, diagnosis and prevention of familial cancer, and surgical treatment of women's cancer. She established the Vaccine and Cervical Cancer Screen (VACCS) study group in 2009, consisting of clinicians and academics from various South African universities. The group has completed two multicentre projects aiming to improve vaccination against and screening for cervical cancer in South Africa and the developing world.

*Snyman LC, Dreyer G, Visser C, Botha MH, van der Merwe FH. The Vaccine and Cervical Cancer Screen project 2 (VACCS 2): Linking cervical cancer screening to a twodose HPV vaccination schedule in the South-West District of Tshwane, Gauteng, South Africa. S Afr Med J 2015;105(3): 191-194. [http://dx.doi.org/10.7196/SAMJ.8888

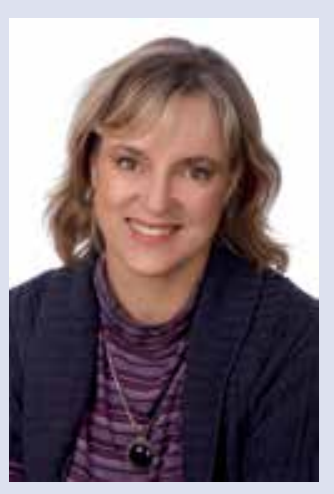

